\title{
Sur la signification conceptuelle de l'intervention des nombres complexes dans la cosmologie
}

\author{
Jean-Jacques Szczeciniarz*
}

Résumé: Le concept de nombre complexe correspond à une certaine forme d'exploration conceptuelle de l'espace; les aspects paradoxaux sous lesquels il s'est manifesté en témoigne. L'élaboration abstraite de cette exploration trouve son aboutissement dans deux opérations conceptuelles mathématiques: la complexification et la compactification qui construit l'espace projectif. Nous esquissons une analyse de celles-ci, et nous la prolongeons dans l'examen des concepts de Penrose (tranformation de Penrose, twisteurs) comme mathématisation conceptuelle de la cosmologie.

Abstract: The concept of the complex number encouters a conceptuel exploration of the space; the paradoxical aspects of it's history bear witness to it. The abstract evolving of this exploration achieved success in two mathematical operations: complexification and compactification that gives birth to the projective space. We are outlining a analysis of it and we extend it by studying the Penrose's concepts (Penrose transformation, twistor) as a conceptual mathematization of the cosmology.

\section{Le nombre complexe}

POURQUOI A-T-ON BESOIN de considérer comme négative une quantité qui se présente à nous comme on ne peut plus positive à savoir un carré? Considérez un carré, nous étendons en une dimension supérieure ce qui se donnait comme un segment de droite, son expression peuple l'espace d'une réalité. Ses éléments constituent et réalisent une portion d'espace. Rappelons-nous les difficultés qu'il y a eu à introduire une grandeur négative. En philosophie. On s'en tire après un long développement de l'algèbre en isolant l'entité positive, on peut l'appeler valeur absolue. Mais le fait d'ajouter du négatif implique une intervention de l'espace avec de nombreuses constructions. L'axe réel m'indi-

* Equipe REHSEIS (UMR 7596) et Université Bordeaux, 37, rue Jacob, 75006 Paris, France, E-mail: szczeciniarz@paris7.jussieu.fr. 
que qu'à partir de la représentation géométrique d'une situation repérée je dois pouvoir régresser en-deçà de l'origine de la constitution première des quantités.

Mais les actes fondateurs de ce type - c'est ce qui fait la difficulté en général, pour les historiens des mathématiques ou les logiciens - les actes fondateurs de ce type concentrent aussi une foule d'autres actes mathématiques. Ainsi le concept de nombre négatif parut-il être construit en liaison avec la constitution d'une nouvelle structure algébrique. Il s'interprète comme un inverse, un opposé, etc. Tous ces concepts - c'est le cas des concepts algébriques - ont un correspondant géométrique. On peut voir géométriquement ausi le zéro comme un point multiple. Il a concentré nombre de paradoxes sur l'identité de la fin et du commencement.

Non seulement une telle situation singularise virtuellement une grande partie de l'activité mathématique, la partie dans le tout, mais elle peut ensuite se déployer sur les autres secteurs des mathématiques par une sorte d'enveloppement en retour.

Ce que nous venons de dire vaut évidemment pour les nombres complexes. Vous connaissez partiellement l'histoire de la constitution des nombres complexes - cette histoire a été rapportée sous des aspects moins connues par D. Flament. Penrose rappelle dans son livre la vie de Cardan. Si les nombres complexes ont été promis à un brillant avenir, c'est pour une part essentielle grâce à Cardan qui en donne le concept et qui ouvre aussi sur la notion de probabilité.

Nous avons bien des difficultés à concevoir l'existence mathématique et même physique de nombres négatifs. Mais la difficulté semblait insurmontable quand il s'est agi de penser comme négative des grandeurs positives. Ecrire $\mathrm{X}^{2}=-1$. Quel statut donner à ce que désigne $\mathrm{X}$ ? D'abord c'est courant en mathématique on se demande comment donner un sens à un résultat qui semble contredire ou bouleverser la définition des objets qui l'ont produit.

La question des quantités "négatives positives", a dû passer par une construction nouvelle de la situation dans l'espace et même par une nouvelle appréhension de l'espace. A chaque fois qu'une telle action est établie elle fait surgir des élemęnts encore plus primitifs - du fonctionnement implicite de notre activité mathématique ou même de pensée.

Elle impose alors que le nombre complexe ainsi constitué représente une quantité dans ce qu'elle peut avoir d'intrinsèquement linéaire et contradictoirement une non-linéarité qui répète l'impossibilité d'un carré négatif. Il réalise en un certain sens une synthèse de la droite et du cercle, pas en même temps mais dans la production d'une temporalité et d'un espace qui suppose la production ou l'expulsion d'une dimension supérieure. Si le nombre imaginaire s'im- 
pose comme entité mathématique ce fut dans cette production de ce mixte impossible qui reprend et analyse cette impossibilté à travers un concept positif. Le nombre imaginaire est donné dans une représentation sous la forme de coordonnées trigonométriques ou dans ce que l'on a appelé le plan Argand-Cauchy qui tienne compte à la fois d'un déplacement mesuré par un angle et d'un déplacement linéaire. L'espace dans lequel il a été construit a laissé l'obstacle de sa construction se déployer sans chercher à le réduire et c'est sous cette forme (module-argument) que nous le manipulons maintenant. Quel a été l'objectif d'une telle construction? Certainement au moins de régresser à une origine minimale de ce que pourrait être une genèse de l'espace. Voir en quoi l'espace est virtuellement du courbe et du droit, comment la virtualité de l'espace est circularité et linéarité.

\section{Cosmologie}

La cosmologie cherche à penser le tout. Non pas tout ce qui est abstraitement, mais elle cherche de façon consubstantielle à se donner une géométrie pour penser le tout. On pourrait presque dire inversement que penser le tout c'est déjà construire une géométrie.

En même temps cette constitution tend toujours à pouvoir nous mettre en position, comme observateurs théoriques, de considérer le tout de l'extérieur. Ceci est certes contradictoire, comme le peut paraître le fait de penser une variété non plongée.

C'est cette difficulté que tente d'élaborer Copernic. Pour ce faire il construit lui aussi des obstacles qui reproduisent pour permettre une pensée les difficultés ainsi cernées. A cet effet il use d'un principe de relativité descriptive: il prend appui de façon positive sur une impossibilité perceptive. Je ne sais de la rive ou du bateau... Cette impossibilité se conjugue chez lui avec une autre qui est liée à la limite de notre perception. Je ne peux percevoir à quelque niveau que ce soit les effets de mon mouvement. Nous ne détectons pas la parallaxe stellaire. Car les distances stellaires sont incommensurables à notre perception. La possibilité du mouvement de la Terre suppose la construction d'un objet non perçu, il faut penser un rapport incommensurable.

Or on sait qu'il est possible grâce aux développements de l'algèbre du $\mathrm{XIX}^{\mathrm{e}}$ siècle de donner une forme mathématique à l'incommensurable. Ainsi construit-on une extenșion de corps pour adjoindre à toutes les opérations qui définissent une action sur les éléments un élément qui était inaccessible. Tel est le cas d'une racine carrée ou nième. 
Une autre opération géométrique nous ménage un accès à l'imperceptible, ou à des éléments rejetés hors de notre perception. Il s'agit de l'ajoût d'un point dit "à l'infini" dans la construction de l'espace de la perspective. On donne un sens positif au fait de se trouver à l'infini. Ce qui suppose d'avoir posé une maîtrise de, une forme d'accès à, ce qui du coup complète "absolument" notre perception ou qui ferme l'espace auquel nous avons accès en nous donnant les moyens de le traiter comme s'il était fini, même si nous en respectons la spécificité.

Deux opérations mathématiques ont ainsi selon nous une signification cosmologique importante, celle de complexifier, de se placer dans un espace géométrique complexe. Elle peut s'effectuer en usant de structures algébriques, on étend le corps des nombres réels. Géométriquement, une fois la conception algébrique assurée, on rend possible par une représentation spatiale une forme de rotation dans la conception et une modalité de perpendicularité aux représentations des nombres réels comme exploration de l'espace. C'est cette veine qu'exploite la Mécanique Quantique.

Deuxième opération, on ferme l'espace par projectivisation: on construit l'espace projectif comme moyen de fermer l'espace dont on accepte l'infinité en établissant la forme de sa maîtrise. Ceci suppose une réélaboration du concept de la sphère, un instant conçu comme obstacle archaïque au dévelopement de notre connaissance.

\section{La Cosmologife et le Complexe}

Il est évident pour qui travaille dans ce domaine que l'ensemble des équations qui rendent compte de la réalité et du cadre de sa constitution est marqué par l'introduction des nombres complexes.

A la lumière des développements précédents je vais me contenter de décrire et de poser certains problèmes que l'introduction de la structure complexe permet présenter.

Il me semble que dès lors que se posent de façon nécessaire des problèmes portant sur l'élaboration - je dirais volontiers ontologique - des objets auxquels nous avons affaire s'impose la nécessité de l'introduction de la structure complexe, sous forme de fonctions d'une part en une variable, d'autre part en plusieurs variables. Et la raison de cette nécessité tient au concept même de nombre complexe dont l'histoire de la formation nous explique la constitution.

Comme cette affirmation reste extrèmement générale, je vais préciser la nature des problèmes ontologiques ainsi posés ou la nature de cette ontologie. L'un des caractères de la structure algébrique que l'on appelle le corps (le 
corps comme structure étant la concentration d'opérations licites que nous sommes en droit d'effectuer sur des êtres mathématiques) des nombres complexes par opposition à celle qui caractérise le corps des réels est l'absence d'ordre au sens archimédien du terme. L'introduction de la circularité dans la linéarité qui fait l'une des substances de $\mathbf{C}$ est nécessaire pour penser les problèmes cosmologiques.

La cosmologie est la science des phénomènes naturels pris dans leur totalité. Ce qui ne veut pas dire science de tout ce qui existe, mais science, de ce qui dans les phénomènes naturels les ordonne en une totalité. N'importe quel phénomène naturel peut intéresser la cosmologie dans la mesure où il est significatif des propriétés du tout. Les recherches des physiciens sur les particules élémentaires ont un intérêt direct pour la cosmologie.

\section{La Renaissance Relativiste et la Signification de l'Introduction du Nombre Complexe}

Einstein, comme l'a rappelé Eisentaedt ${ }^{1}$, a posé au début du $\mathrm{XX}^{\mathrm{e}}$ siècle le problème cosmologique en des termes tout à fait nouveaux. Einstein a été amené à résoudre le problème de la gravitation, à le traiter en donnant une interprétation géométrique des phénomènes physiques de l'inertie et de la gravité. Il pense que la géométrie de l'espace et du temps physique est déterminée par les propriétés de la matière-énergie de la région où l'on se place. Lois physiques et axiomes géométriques sont de ce fait étroitement liés.

Prenons le problème de la gravitation.

Pourquoi une planète, image idéale d'une particule libre ne se déplacet-elle pas en ligne droite? C'est la solution newtonienne de la force d'attraction solaire. On connaît les difficultés de l'interprétation physique d'une telle loi, car elle s'est présentée d'abord comme une action à distance. D'où le fait qu'Einstein ait décrit le phénomène de la gravitation à l'aide d'un espace-temps à courbure.

S'est déplacée en physique et en cosmologie - peut-être n'ont elles fait que la développer - la détermination géométrique de notre physique et peutêtre de notre compréhension générale des phénomènes de la nature.

Sans entrer dans le détail et la profondeur du principe de Mach, on peut rappeler que sa signification essentielle est de poser la détermination de la géo-

1 Cf. Dans ce volume. 
métrie de l'espace-temps par son contenu matériel. Une propriété physique se traduit par une propriété géométrique, une masse par une propriété de courbure.

Ceci est bien connu, mais je voudrais insister brièvement sur le fait que s'il en est ainsi c'est aussi parce que la géométrie construite de cette façon est dévelopée sur le mode de l'intrinsincéité. On sait montrer - c'est un des aspects de la théorie de Gauss - que la courbure gaussienne d'une surface est intrinsèquement déterminée.

Autrement dit la géométrie dont il est question est une géométrie qui a "densifié" ses objets, et qui par certains côtés a fait entrer la physique qu'elle permet de conceptualiser dans la mise en forme de ses propres concepts. La géométrie organise depuis l'intérieur la physique qu'elle fait comprendre et appréhender. En termes kantiens, je dirais volontiers qu'elle ne met pas en œuvre seulement des principes mathématiques mais aussi des principes dynamiques. Elle anticipe dans sa forme sa propre physique.

Soulignons que cette géométrie construit aussi les formes de son objectivité, qui s'exprime dans le calcul tensoriel: celui-ci montre qu'une loi physique ne doit pas dépendre du référentiel choisi pour l'écrire.

Il existe donc un être mathématique particulièrement adapté à la description de et même à la réflexion sur l'espace temps qu'on appelle un tenseur. Je ne vais en donner qu'une description conceptuelle, qualitative.

Nous cherchons d'abord des moyens d'effectuer des mesures sur une surface. Ce qui nous en rend capable est ce que l'on appelle une métrique ou manière de mesurer, donnée par le carré de la distance entre deux points- elle nous présente les moyens d'une progression dans des directions qui "couvrent" infinitésimalement la surface. Elle doit être invariante en un certain sens. On doit pouvoir mesurer de la même façon quel que soit le lieu de la mesure. Ou encore la métrique doit être invariante par changement de coordonnées. D'où la forme classique que vous connaissez tous de cette métrique avec les $g^{\mu \nu}$

La condition nécessaire et suffisante pour qu'un espace soit sans courbure est qu'il existe au moins un système de coordonnées dans lequel les g $\mu \nu$ soient des constantes. Sans y insister on voit que c'est dans la forme des équations que se reflète la propriété géométrique. Ce qu'a recherché Einstein c'est un tenseur géométrique, le plus général qui permette de définir la métrique c'està-dire la géométrie de l'espace-temps, entièrement déterminé par le contenu matériel du milieu.

Un tenseur est une sorte d'opérateur conceptuel qui donne par la forme de variation de ses éléments la nature de l'objet qu'il décrit.

Il est remarquable qu'il concentre les propriétés qui le plus souvent sont construites à partir de différentiation. Parmi ces tenseurs il en est un, le tenseur 
de métrique. Dans des conditions données on peut choisir les coefficients de cette métrique de façon relativement arbitraire. En particulier, le fait de choisir une symétrie sphérique de l'espace permet de résoudre toutes les équations dites d'Einstein qui doivent permettre de déterminer la métrique.

Le caractère arbitraire de la métrique autorise le signe négatif devant les termes spatiaux. On peut ainsi différencier les termes spatiaux et le terme temporel, par le signe des coefficients. Mais si on fait une coupe avec un temps nul, on a affaire à une somme de coefficients négatifs. On a ainsi une hypersurface complexe qui nous décrit une intervalle purement imaginaire.

On peut interpréter ce résultat comme l'impossibilité physique d'arrêter l'écoulement du temps, sous peine de nous retrouver dans un espace qui n'existe pas. C'est la façon dont les concepts de nombre imaginaire nous font connaître le temps qui impose cette interprétation.

\section{La Complexification}

Considérons maintenant de façon générale la métrique de Minkowski. L'espace-temps est un espace réel à quatre dimensions de points $x_{\text {. }}=\left(\mathrm{x}_{0}, \mathrm{x}_{1}, \mathrm{x}_{2}\right.$, $\mathrm{x}_{3}$ ) muni de la métrique "hyperbolique"

$$
|| x||^{2}=x_{0}^{2}-x_{1}^{2}-x_{2}^{2}-x_{3}^{2}
$$

Avec $\mathrm{x}_{0}$ figurant le temps On sait que la vitesse des signaux est inférieure à celle de la lumière, $c$, prise égale à 1 . L'équation $\|\mathrm{x}\| \|^{2}=0$ définit dans l'espace réel de Minkowski, $M$, un cône de lumière de sommet $x=0$. L'intérieur de ce cône $\left\{\mathrm{x}_{1}{ }^{2}+\mathrm{x}_{2}{ }^{2}+\mathrm{x}_{3}{ }^{2}<\mathrm{x}_{0}{ }^{2}\right\}$ se décompose en deux nappes appelées cône du futur $\left(\mathrm{x}_{0}>0\right)$ et cône du passé $\left(\mathrm{x}_{0}<0\right)$. Ces cônes sont composés de tous les points avec lesquels, en vertu du postulat fondamental, peut communiquer le point $\mathrm{x}=0$ dans le futur et dans le passé. Il est apparu nécessaire de complexifier l'espace $\mathrm{M}$. On l'a donc plongé dans $\mathbf{C}^{4}$ (espace complexe de dimension 4) en tant que sous-espace réel $\mathbf{R}^{4}(\mathrm{x})$ et complété avec les points $z=x+$ iy, pour lesquels $\mathrm{y}_{1}{ }^{2}+\mathrm{y}_{2}{ }^{2}+\mathrm{y}_{3}{ }^{2}<\mathrm{y}_{0}{ }^{2}$. Considérons les cônes $C_{+}$et $C$ - $\left\{\mathrm{y} \in \mathbf{R}^{4}, \mathrm{y}_{0}{ }^{2}-\mathrm{y}_{1}{ }^{2}\right.$ $\mathrm{y}_{2}^{2}-\mathrm{y}_{3}{ }^{2}>0, \mathrm{y}_{0}>0$ et $\left.\mathrm{y}_{0}<0\right\}$. Puis considérons les domaines tubulaires au-dessus d'eux. $M_{c}=\left\{z=x+i y, x \in \mathbf{R}^{4}, y \in C_{+}\right.$et $\left.C_{-}\right\}$. Qu'est-ce qu'un tube?

C'est une exploitation géométrique de la structure complexe, dans le multidimensionnel. Un nombre complexe est représenté suivant l'axe réel et l'axe perpendiculaire à cet axe, l'axe imaginaire pur; il s'agit de transposer sur plusieurs dimensions à la fois cette structure prise initialement sur deux dimensions. Ce qui s'écrit alors $\mathrm{T}=\mathrm{B}(\mathrm{x})+i \mathbf{R}^{\mathrm{n}}(\mathrm{y})$. C'est là ce que nous appliquons à 
l'espace réel de Minkowski. Nous obtenons un domaine tubulaire au-dessus de ces cônes qui est l'espace de Minkowski complexifié. $M^{c}=M^{c}+U M U M^{c}-M$ l'espace réel de Minkowski est la frontière distinguée des domaines tubulaires complexes $M_{c}$

La complexification permet d'explorer le fait de mesurer en le considérant sous l'aspect de ces formes d'impossibilité mathématisées que permettent les extensions complexes. Ou bien inversement il est possible de donner une signification à la forme d'extension de notre manière d'appréhender mathématiquement, cette forme d'extension correspondant à une extension par les nombres complexes.

La théorie des torseurs dont les origines s'expliquent pour une grande part par les paradoxes EPR, donne des descriptions des notions physiques fondamentales. Je vais simplement vous donner l'exemple des équations de Maxwell.

Elle propose une description non locale de l'espace-temps dans laquelle les rayons lumineux sont représentés par de simples points. C'est cette non-localité spatio-temporelle qui la relie à la non-localité quantique des situations EPR. Elle repose fondamentalement sur les nombres complexes et la géométrie qui leur est associée.

De façon générale, la métrique de Minkowski, métrique hyperbolique, sépare l'espace réel sur lequel elle est déployée en différents cônes. Faisons une remarque sur ces fameux cônes. Il me semble qu'ils remontent à une tradition d'utilisation de forme de généralisation (Desargues) des figures géométriques pour décrire et construire même les formes de notre subjectivité. C'est Leibniz qui a développé ce mode de construction, usant du sommet du cône pour placer le regard synthétisant de la monade. Le passage d'une monade à une autre étant en tout cas impossible depuis l'intérieur de l'une d'elle - elle est sans portes ni fenêtres.

Ajoutons pour les géomètres le fait que le cône décrit un mode de déploiement où le déplacement vertical - dans une direction - contrôle le déplacement qui lui est perpendiculaire et reste circulaire. C'est la raison de la richesse de ses sections. Le cône est à même grâce à sa géométrie de décrire des formes de clôture subjective - comme la sphère - mais aussi de situer ces subjectivités les unes par rapport aux autres. La métrique hyperbolique installe ces formes de synthèse et de séparation. Vous avez les cônes de lumière qui sont donnés par les équations du cône et les événements, quant à eux, vous sont donnés 'comme événements particuliers (présent, passé et futur) dans chaque cône.

Quelle est la signification de cette situation monadologique? Eddington qui fait l'objet de la thèse complémentaire de Jacques Merleau-Ponty déve- 
loppe des idées similaires. (C'est aussi le point de vue de Milne). Sur le plan de la conception "cosmologique" nous sommes des suites d'événements monadologiques dont aucune ne peut interférer, mais qui contribuent de leur point de vue à la beauté du tout (Leibniz).

Passons maintenant à la complexification de cet espace de Minkowski. Il s'agit de voir $\mathbf{R}^{4}$ comme une partie de $\mathbf{C}^{4}$ Cette opération est mathématiquement très puissante. On colle au-dessus de ces cônes réels des tubes qui permettent des les voir comme une partie imaginaire de ce grand espace complexe.

On remarque que toujours dans une telle construction subsiste la forme géométrique cette fois rendue bien abstraite, qui permet à travers sa propre géométrie de réorganiser l'espace. Vous avez donc un cône au-dessus duquel vous collez un voisinage tubulaire, un tube. Par exemple une bande de $\mathbf{C}$ est un tube. Comme on est ici dans plusieurs variables complexes (à la fois) il faut trouver un moyen géométrique de travailler cette pluralité. Or il est des formes géométriques qui permettent - quand elles sont abstraites - ce déploiement, sorte de repliement éclaté des formes intuitionnables sur celles qui ne le sont point. La représentation passe ici par la forme abstraite de l'équation qui répète littéralement la construction visible du tube (cylindre).

Lorsque l'on a complexifié l'espace de Minkowski, on a gagné une nouvelle situation de celui-ci. Comme $\mathbf{R}$ dans $\mathbf{R}(\mathrm{i})$. L'espace réel de Minkowski constitue alors la portion commune des frontières des domaines tubulaires au-dessus des cônes.

Je donne encore un exemple de complexification. Pauli a eu l'idée de représenter les points de l'espace réel $M$ par des matrices. Ces matrices sont hermitiennes et ont leurs termes construits de telle manière que leur déterminant soit le cône sur lequel on travaille: $\|x\|^{2}$. Pour ce faire on a été obligé de passer par le complexe, qui sert ensuite à nous ramener dans l'espace réel.

$$
X=\left(\begin{array}{ll}
x_{0}+x_{1} & x_{2}+i x_{3} \\
x_{2}-i x_{3} & x_{0}-x_{1}
\end{array}\right) ; \text { On constate facilement que det } X=\|x\|^{2}
$$

Lorsque l'on prolonge à $\mathbf{C}^{4}$ la tranformation de Pauli

$$
L:\left(\begin{array}{ll}
z_{0} & z_{1}, z_{2}, z_{3}
\end{array}\right) \rightarrow\left(\begin{array}{cc}
z_{0}+z_{1} & z_{2}+i z_{3} \\
z_{2}-i z_{3} & z_{0}-z_{1}
\end{array}\right)
$$

on constate qu'elle agit sur les domaines tubulaires de manière à les envoyer dans un autre domaine de $\mathbf{C}^{4}$ qui soit un cadre de travail, ce qu'on appelle un polyplan 
supérieur généralisé (demi-plan de Poincaré). On a représenté les points de $\mathbf{C}^{4}$ par des matrices 2-2. Soit $Z^{*}=\left(\mathbf{z}_{j \mathrm{k}}^{-}\right)$la transposée conjuguée de la matrice $Z$; on pose

$$
\operatorname{Im} Z=1 / 2 i\left(Z-Z^{*}\right)
$$

On appelle demi-plan supérieur généralisé le domaine

$$
\begin{gathered}
H=\left\{Z \in C^{4}: \operatorname{Im} Z>0\right\} . \text { Soit } \\
H=\left\{Z \in C^{4} ;\left(\begin{array}{l}
y_{11} \frac{z_{12}=\overline{z_{21}}}{2 i} \\
\frac{z_{21}=\frac{z_{121}}{2 i}}{y_{22}}
\end{array}\right)>0\right\}
\end{gathered}
$$

On constate que cette matrice est hermitienne, les inégalités et égalité

$$
\begin{aligned}
& \mathrm{y}_{11}>0, \quad \mathrm{y}_{11} \mathrm{y}_{22}-\frac{1}{4}\left|z_{12}-\bar{z}_{21}\right|^{2}>0 \\
& \mathrm{y}_{11} \mathrm{y}_{22}-\frac{1}{4}\left|\mathrm{z}_{12}-\bar{z}_{21}\right|^{2}=0 \\
& \mathrm{y}_{11}>0, \quad \mathrm{C}=\left\{\mathrm{y}_{11}{ }^{2}-\mathrm{y}_{221}{ }^{2}-\mathrm{y}_{12}{ }^{2}-\mathrm{y}_{21}{ }^{2}>0\right\}
\end{aligned}
$$

définissant le demi-plan supérieur et sa frontière.

Que signifie cette représentation? C'est d'abord une simple organisation des coordonnées. Mais c'est aussi une façon d'encadrer les éléments qui sont placés en position particulière accessible sur cette matrice (sur la diagonale principale les éléments sont réels et les symétriques par rapport à cette diagonale sont conjugués).

On peut remarquer encore ce que signifie cette concentration conceptuelle, la conjugaison des éléments dans le corps des complexes est une façon de conserver l'extension dans $\mathbf{C}$.

$$
\text { Si } Z=\left(\begin{array}{ll}
z_{00} & z_{01} \\
z_{10} & z_{11}
\end{array}\right)
$$

est la représentation matricielle des points de $\mathbf{C}^{4}$ on a $\operatorname{Im} Z=$

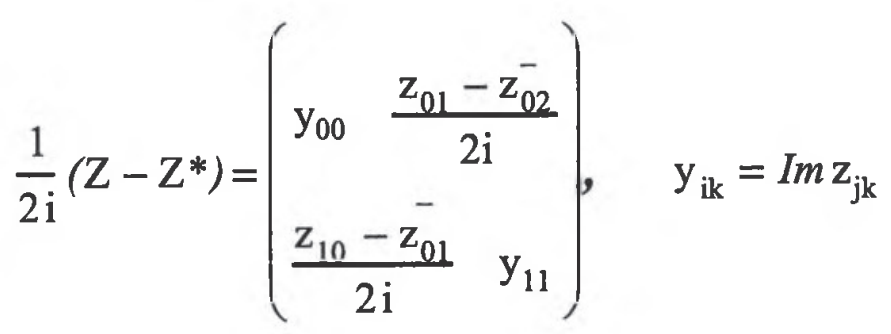


On a comme vu ci-dessus un matrice hermitienne et les signes $>$ et $<$ expriment le fait que cette matrice soit positive ou négative. On remarque que l'espace $M$ se transforme par cette application $L$ en un ensemble de matrices hermitiennes $Z$ pour lesquelles $\operatorname{Im} Z=0$, soit en un plan $\left\{y_{00}=y_{11}=0, z_{01}=z_{10}\right\} \subset \mathbf{C}^{4}$, de dimension réelle 4 . On ne fait plus de distinction entre $M^{c}$ et $L\left(M^{c}\right)$.

\section{La compactification}

On est amené à étudier ce qui se passe très loin de l'origine des coordonnées. On cherche donc à achever $\mathbf{C}$. On lui adjoint ce qu'on appelle un point à l'infini. Comme on peut être amené à adjoindre des droites de l'infini à des espaces de dimension plus grande. On a alors affaire à une fermeture de l'espace, qui nous permet de ramener les problèmes à l'infini à des problèmes qui se cherchent sur des parties finies. Cette opération se nomme opération de compactification.

Cette opération de compactification est une façon de traiter de l'infini comme s'il était fini. C'est de fait en un certain sens l'exploitation de la nature conceptuelle de ce qu'est une sphère, une façon de conceptualiser la sphère. L'univers a toujours été conçu comme sphérique, la forme du tout a longtemps été introduite dans la réflexion philosophique comme celle de la sphère. On peut affirmer que l'opération de compactification ici, le passage au projectif est aussi une des formes que peut prenrde la synthèse. Dans une réflexion cosmologique elle se manifeste sous deux formes

D'une part du côté du tout: il faut pouvoir se donner une représentation du tout et nous avons les modèles de type sphérique ou projectif du tout. D'autre part et c'est souvent méconnu, il y a ce que j'appellerai une réflexion locale cosmologique: le fait que l'on utilise comme outil des formes globales cosmologique comme si le tout ressurgissait - comme le montre Hegel - dans ses élements ou dans ses parties. C'est le cas de l'opération de compactification généralisée.

Penrose a inventé ce que l'on pourrait appeler une compactification généralisée. Dans un premier temps on considère donc le compactifié d'un plan complexe, soit $\mathbf{C}$ auquel on ajoute un point à l'infini. Les espaces projectifs sont tous construits de cette manière.

On a ainsi $\mathbf{P}^{3}$ qui l'espace projectif de dimension complexe trois. Qui est obtenu à partir de la compactification de $\mathbf{C}^{3}$. On peut l'obtenir en identifiant des points antipodiques de la sphère $\mathbf{S}^{3}$ réelle. Le but est de travailler sur des points de cet espace, qui sont alors des objets géométriques de dimension 
appropriée. Une droite de $\mathbf{P}^{3}$ c'est un espace projectif de dimension 1. Ou encore un cercle sur la sphère $\mathbf{S}^{3}$ Ou encore un plan complexe compactifié.

Penrose considère toutes les droites de $\mathbf{P}^{3}$ à la fois. Cet objet géométrique est muni de structures très riches. Et il est compact, il récolte la compacité de ses éléments. On peut parfaitement dire ce que sont ces structures et ce que sont les éléments. Ce que fait Penrose c'est d'inclure le complexifié de Minkowski dans un tel espace compact.

Par cette opération il colle à l'infini de l'espace réel de Minkowski le cône réel de $\mathbf{R}^{4}$

Cette opération est décrite d'abord matriciellement, comme une condition sur le déterminant de la matrice qui représente un point du grand espace compact. Il faut remarquer que Minkowski hérite du résultat de l'opération de compactification de l'espace constitué de toutes les droites complexes de $\mathbf{P}^{3}$ Mais que l'opération reste structurellement la même. On réunit à un espace fini, un autre espace qui était à l'infini. Cette "réunion" consiste à rendre possible dans la figure même la considération de points à l'infini.

Il est remarquable que cette partie à l'infini nous soit donnée par des conditions portant sur les coordonnées de l'espace que l'on construit. Il existe une manière de se déplacer dans un espace ainsi donné qui laïcise le point à l'infini. Il y aura une partie d'espace où il se trouve qui nous donnera accès à lui. L'opération d'ajoût de parties à l'infini est une opération dont les effets se traduisent partout dans la géométrie de l'espace projectif construit.

\section{Remarques sur l'idée de transformation intégrale à la base de la théorie des twisteurs}

L'idée de Penrose est donc d'utiliser de façon systématique cette compactification généralisée. Il s'agit de replier l'espace compact sur ses éléments. A cause de l'importance des structures diverses qu'il nous livre. A chaque point de cet espace on peut faire correspondre une droite projective. On passe donc de l'espace de Minkowski projectif complexe (compactifié complexifié) à l'espace projectif de dimension trois qui lui est sous-jacent. Les points de cet espace sont appelés twisteurs. Cette méthode générale consiste à reprivilégier au sein de la structure générale, comme ensemble en tant que tel de droites de $\mathbf{P}^{3}$, une droite correspondant à un point quelconque de l'ensemble général. En particulier on peut ainsi observer les points de l'espace de Minkowski. 
A un point on peut associer une droite. Remarquons bien qu'un point de l'espace dont on part est représenté par des conditions sur l'ensemble des droites de $\mathbf{P}^{3}$ en tant que tel. Il faut bien noter qu'une droite de $\mathbf{P}^{3}$ est une droite complexe projective notée $\mathbf{P}^{1}$ que l'on appelle sphère de Riemann. Une sphère de Riemann est un espace complexe de dimension1, $\mathbf{C}$ que l'on a complété par un point à l'infini comme vu. Ces conditions se traduisent par des expressions matricielles.

On munit cet ensemble, appelé grassmannienne, d'une structure de variété complexe. Un point de cette variété, donc une droite projective peut s'écrire: $\tilde{Z}=\left(\begin{array}{l}E \\ Z\end{array}\right)=\left(\begin{array}{cc}1 & 0 \\ 0 & 1 \\ z_{00} & z_{01} \\ z_{10} & z_{11}\end{array}\right) \begin{aligned} & \text { l'ensemble de ces matrices est la partie affine de cette } \\ & \text { variété. }\end{aligned}$

Les points de la variété grassmannienne se représentent par des classes de $4 \times 2$ - matrices $Z=\left(\begin{array}{l}Z_{1} \\ Z_{2}\end{array}\right)$ deux matrices $Z$ ' et $Z$ étant équivalentes s'il existe une matrice $C$ d'ordre 2 telle que $Z^{\prime}=Z C$. Les points de la partie affine de cette variété se représentent par des matrices $Z$ avec $\operatorname{det} Z_{1} \neq 0$. Car on retrouve ainsi facilement la forme $\tilde{Z}$. La condition $\operatorname{det} Z_{1} \neq 0$ possède une interprétation particulièrement intéressante. Nous avons inclu l'espace complexe de Minkowski, $\mathrm{M}^{\mathrm{c}}$, dans la variété grassmannienne qui est compacte. De la même façon que l'on compactifie $\mathbf{C}$ en ajoutant un élément à l'infini $\{\infty\}$. les points de la partie affine de la grassmannienne auxquels on ajoute cette condition sont les points à l'infini de la variété. Ce sont ces points que l'on colle à l'infini de $\mathbf{C}^{4}$ par cette compactification. Si l'on revient en réel, à l'espace réel $M$ on ajoute l'ensemble des matrices pour lesquelles det $X=\mathrm{x}_{0}{ }^{2} \mathrm{x}_{1}{ }^{2}-\mathrm{x}_{2}{ }^{2}-\mathrm{x}_{3}{ }^{2}=0$, on colle à l'infini de cette façon un cône de $\mathbf{R}^{4}$ On ne s'intéresse pas à $\mathbf{C}^{4}$ tout entier mais à la partie $\mathrm{M}^{\mathrm{c}}$. On met en évidence cette partie de la manière suivante.

Comme la transformation de Pauli envoie les domaines tubulaires $\mathrm{MC}+$ et - dans les demi-plans supérieurs et inférieur $\mathrm{H}+$ et - i.e. $\{\mathrm{Z} ; \operatorname{ImZ}>0$ et $<0\}$ on construit les domaines

$\tilde{\{Z} ; \operatorname{Im} \tilde{Z}>0$ ou $<0\}$. Il suffit de construire l'application $\Phi$ telle que 
$\Phi(\tilde{Z})=\left(\mathrm{E}, \tilde{Z}^{*}\right)\left(\begin{array}{cc}0 & -\mathrm{iE} \\ \mathrm{iE} & 0\end{array}\right)\left(\begin{array}{l}\mathrm{E} \\ \mathrm{Z}\end{array}\right)=-\mathrm{i}\left(\tilde{\mathrm{Z}}-\tilde{Z}^{*}\right)=2 \operatorname{Im} \tilde{\mathrm{Z}} \quad \mathrm{Z}^{*}$ est la transposée conjuguée de Z. On obtient donc $\tilde{\mathrm{M}}^{\mathrm{C}+}$ et ${ }^{-}$qui sont les compactifiés de $\mathrm{M}^{\mathrm{c}}+$ et

On a de même $\tilde{M}=\{\tilde{Z} \in \mathrm{G}(3,1) ; \phi(\tilde{Z})=0\} ;(\mathrm{G}(3,1)$ étant la variété grassmannienne, ensemble des droites complexes de $\mathbf{P}^{3}$.

Les points de cette variété peuvent donc être interprétés comme des droites complexes ou comme des plans de $\mathbf{C}^{4}$ (dimension complexe 2 dans un espace de dimension complexe 4). Pour les points de la partie affine représentables par les matrices $\tilde{Z}$, les plans sont de la forme

$\mathrm{w}=\lambda\left(\begin{array}{l}1 \\ 0 \\ \mathrm{z}_{00} \\ \mathrm{z}_{10}\end{array}\right)+\mu\left(\begin{array}{l}0 \\ 1 \\ \mathrm{z}_{01} \\ \mathrm{z}_{00}\end{array}\right)$ w étant un vecteur colonne $\left(\mathrm{w}_{\mathrm{i}}\right)$ et $\lambda \mu$ des paramètres com-

plexes. On obtient facilement les équations développées d'une droite projective correspondant à un point $\tilde{Z}$

Penrose passe de l'espace complexe de Minkowski à un espace projectif complexe dont les points s'appellent des twisteurs. La tranformation de Penrose associe à tout point

$\tilde{\mathrm{Z}} \in \tilde{\mathrm{M}}^{\mathrm{c}}$ la droite projective correspondante $\quad 1 \subset \mathbf{P}^{3}(\mathbf{P T})$,

ou encore la sphère de Riemann correspondante. On munit ensuite l'espace des twisteurs d'une forme hermitienne. On voit aisément que cette forme définit dans $\mathbf{P}^{3}$ (= PT) une hypersurface réelle $\mathbf{N}$ (dimension réelle 5) qui partage $\mathbf{P}^{3}$ en deux domaines $\mathrm{D}+$ et - .

$$
\phi(\mathrm{w})=\mathrm{w}^{*} \phi \mathrm{w}=\mathrm{i}\left(\mathrm{w}_{0} \overline{\mathrm{w}}_{2}+\mathrm{w}_{1} \overline{\mathrm{w}}_{3}-\overline{\mathrm{w}}_{0} \mathrm{w}_{2}-\overline{\mathrm{w}}_{1} \mathrm{w}_{3}\right)=-2 \operatorname{Im}\left(\mathrm{w}_{0} \overline{\mathrm{w}}_{2}+\mathrm{w}_{1} \overline{\mathrm{w}}_{3}\right),
$$

cette hypersurface réelle $\mathrm{N}=\left\{\mathrm{w} ; \operatorname{Im}\left(\mathrm{w}_{0} \overline{\mathrm{w}}_{2}+\mathrm{w}_{1} \overline{\mathrm{w}}_{3}\right)=0\right\}$. On montre que la transformation de Penrose associe aux points $\tilde{\mathrm{Z}} \in \tilde{\mathrm{M}}^{\mathrm{c}}{ }_{ \pm}$des droites entièrement contenues dans les domaines $D_{ \pm}$des twisteurs positifs et négatifs et aux points de $\mathrm{M}$ des droites de l'hypersurface $\mathrm{N}$ des twisteurs nuls.

Dans l'espace $\mathbf{C}^{4}$ on peut déterminer comme précédemment des cônes, dits cônes de lumière complexes. Ceux-ci sont déterminés à partir de condition $\mathrm{d}^{\prime}$ intersection des droites images de la transformation de Penrose. Si $\mathrm{p}\left(\mathrm{Z}^{0}\right)=\mathrm{l}_{0}$ 
est l' image de $Z^{0}$ et les images $p(Z)=p\left(Z^{0}+V\right)$ celles des points $Z^{0}+V$, couperont $l_{0}$ si et seulement si det $V=0$. L'hypersurface complexe

$$
\mathrm{C}_{\mathrm{z}_{0}}=\left\{\mathrm{Z}=\mathrm{Z}^{0}+\mathrm{V} ; \operatorname{det} \mathrm{V}=0\right\} \text { s'appelle cône de lumière complexe. }
$$

Et sur ce cône on peut différencier deux familles de plans différents. La transformation de Penrose agit sur ces plans. Leur associant pour les uns un point de $\mathbf{P}^{3}$ (= PT, twisteurs) et pour les autres un plan de l'espace des twisteurs, un point de l'espace conjugué des twisteurs $\left(\mathbf{P}^{3}\right)^{*}$

On restreint le cône de lumrère complexe à sa partie réelle, on appelle cône de lumière réelle. Les points de ce cône sont situés à une distance nulle de son sommet, d'où son nom de cône d'isotropie.

Le cône réel est composé de droites réelles appelées rayon de lumière.

Vous voyez comment un twisteur est l'équivalent ponctuel et non local d'un plan du cône de lumière. Et l'image d'un point du rayon de lumière par la transformation de Penrose est une droite de l'ensemble des twisteurs nuls. Qu'est-ce qu'un twisteur nul? C'est un point qui est situé sur une certaine surface réelle de l'espace des twisteurs. Dans cet espace on introduit en effet des instruments qui permettent d'ordonner les éléments.

On s'est donné les moyens d'observer dans un autre espace mathématique les cônes qui concentrent les événements.

La tranformation de Pauli complexe qui permet d'écrire en coordonnées sur la variété grassmannienne l'application est ce qu'on appelle aussi la relation d'incidence qui établit cette correspondance ou transformation de Penrose. On peut écrire cette relation à l'aide de la notation spinorielle.

On a commencé à bâtir une théorie quantique des twistors. On se donne une fonction d'onde des twisteurs à valeurs complexes définie sur l'espace des twisteurs. Penrose explique que n'importe quelle fonction $\mathrm{f}(\mathrm{Z})$ n'est pas a priori une fonction d'onde mais utilise la propriété d'holomorphie pour caractériser une telle fonction. De la même façon il donne une description de $f(Z)$ dans l'espace-temps. Elle s'obtient au moyen d'une intégrale de contour et là encore la contrainte d'holomorphicité des champs de twistors code pour les équations du champ.

C'est pourquoi cette méthode géométrique permet ausi de résoudre et de classifier les équations de l'électromagnétisme et de l'optique géométrique. En associant à chaque droite donnée par l'application dans les twisteurs une opération sur celle-ci qui permette de donner des équations de Maxwell une représentation: on effectue une intégration le long de ces droites. Cette fois la transformation qui donne des solutions des équations différentielles une re- 
présentation intégrale est une méthode absolument générale de géométrie intégrale. On peut comprendre qu'une opération d'intégration anticipe sur des solutions d'équations différentielles.

Il est simple de remarquer qu'une intégration c'est d'abord une forme d'anticipation, et qu'elle renoue avec les anticipations que Kant avait tenté de thématiser dans l'analytique de la Critique de la Raison Pure.

Pourquoi un tel cadre pour les équations de Maxwell, qui peut être développé encore pour d'autres équations différentielles importantes de la physique mathématique. Je dirais sans l'expliciter que c'est parce que cette géométrie s'est développée comme cadre de conception des formes de solutions aux limites. C'est le mode de compactification complexe qui explique la puissance de cette classification.

\section{Quelle en est la place dans la cosmologie?}

Comme je l'ai souligné il s'agit d'une des formes de totalisation donnée à travers cette opération centrale de compactification.

Il en est de même pour les modèles cosmologiques qui sont à la base de la recherche de l'évolution de la métrique globale de l'univers.

Vous comprenez bien qu'il serait facile de dire que comme il faut anticiper sur l'évolution de l'univers, nous avons affaire à une sorte de système différentiel total. Que nous donnent les équations d'Einstein. C'est plus difficile. Il faut pourtant une sorte de principe anthropique. Par lequel nous remontons aux questions initiales de la métaphysique.

"Le résultat fondamental de Penrose et Hawcking consiste en un théorème d'existence nécessaire de singularité. Ils démontrent qu' une singularité doit être présente pour autant que soient vérifiées trois conditions. La première porte sur la structure causale de l'univers; la seconde sur le contenu énergétique; la troisième exprime que la gravité doit être suffisamment intense."2

2 Lachièze Rey M., Présentation de "La nature de l'espace et du temps". S. Hawking R. Penrose, trad.

F. Balibar, NRF, 1996. 
Si comme le souligne le même auteur on peut considérer l'espace-temps comme tissé par l'ensemble des géodésiques, connaître ces géodésiques et la structure qu'elles dessinent cela permet de savoir quelle région de l'univers peut influencer telle autre. C'est ainsi que l'on a affaire la structure causale de l'univers. Selon la relativité générale c'est le contenu énergétique de l'univers qui détermine(au moins partiellement) la structure de l'espace-temps.

D'après l'énoncé de Penrose l'univers en expansion entraine la présence d'une singularité dans le passé. C'est pour examiner dans un cadre mathématico-théorique suffisamment puissant la nature de ces singularités que Penrose a developpé sa théorie twistorienne. Il en parle comme d'une idéologie.

La structure de l'univers est décrite à l'aide d'un concept mathématique appellé tenseur de Riemann de l'espace-temps lié à sa courbure. Penrose le décompose en deux parties qui s'additionnent le tenseur de Ricci et le tenseur de Weyl. Selon Penrose la partie "Ricci" exprime une structure globale et relatuvement régulière et la partie "Weyl" rend compte des éventuelles irrégularités ${ }^{3} \mathrm{Le}$ big bang extrêmement régulier se décrit donc avec tenseur de Weyl nul. L'hypothèse de l'annulation du tenseur de Weyl est liée au nom de Penrose ${ }^{4}$

Comme la courbure de Weyl devait valoir zéro aux singularités du passé et que la variété d'espace-temps est quasi conformément plate la théorie des twistors y est très bien adaptée. On se trouve dans la situation où l'holomorphie exprime les équations du champ. Mais Penrose explique qu'il veut suivre l'idéoléogie twistorienne même si la description se complique avec l'envahissement de la courbure de Weyl c'est-à-dire avec l'écoulement du temps. Il ajoute même que c'est l'idéologie twistorienne qui le fait pencher pour un modèle d'univers ouvert, modèle d'univers avec $\mathrm{k}<0$. $\mathrm{K}$ mesure la force du champ de gravitation sur l'horizon des événements ${ }^{5}$ Sa préférence pour $\mathrm{k}<0$ vient de ce que le modèle twistorien holomorphe continue de fonctionner (le groupe de symétrie de la singularité initiale est holomorphe).

La question qui reste encore ouverte est de savoir en l'occurrence si la logique intrinsèque de la théorie permet de trancher sur la pertinence d'un modèle physique. A ce niveau de généralité, la réponse ne peut qu'être idéologique comme l'indique Penrose. Cependant il devient clair que la structure mathématique de la théorie joue un rôle heuristique dont nous avons déjà vu les raisons.

3 Ibidem, p.75 et Merleau-Ponty, J. Cosmologie du XXè siècle pour les modèles classqiues, Friedmann Lemaître, Robertson et Walker.

4 Lachèze-Rey, ibidem.

5 Hawqking et Penrose, ibid., p. 63 et ss. 


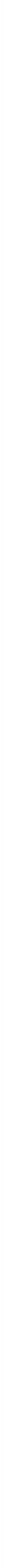

